\title{
A WEB PROCESSING SERVICE FOR GNSS REALISTIC PLANNING
}

\author{
Bianca Federici $^{\mathrm{a}}$, Davide Giacomelli ${ }^{\mathrm{b}}$, Domenico Sguerso ${ }^{\mathrm{a}}$, Alfonso Vitti ${ }^{\mathrm{b}}$, Paolo Zatelli ${ }^{\mathrm{b}}$ \\ a DICAT - Dipartimento di Ingegneria delle Costruzioni, dell'Ambiente e del Territorio \\ Università degli Studi di Genova \\ 16145 Genova, ITALY \\ (bianca.federici, domenico.sguerso)@unige.it \\ ${ }^{\mathrm{b}}$ DICA - Dipartimento di Ingegneria Civile e Ambientale \\ Università degli Studi di Trento \\ 38123 Trento, ITALY \\ (alfonso.vitti, paolo.zatelli)@unitn.it
}

Commission IV/5

KEY WORDS: GIS, Planning, GPS/INS, Internet, Services

\begin{abstract}
:
The spread presence of GNSS Permanent Station Networks makes satellite surveys simpler, quicker and cheaper, and the achievement of high precisions easier. However, the result of a GNSS campaign benefits from an accurate planning, especially in presence of natural or artificial obstructions that make satellite observations difficult. Most of the GNSS office suites provide planning tools to analyze the satellite's visibility; visibility plots, sky plots and DOP charts are commonly built from the GNSS almanac. They usually allow planning on single points, taking into account the obstructions due to terrain morphology or buildings by hand-drawing polar diagrams after a visit of each location to be surveyed. This task can be made fully automatic by using a digital surface model to build the obstruction plot and the almanac to evaluate satellites' positions, hence their visibility, in a GIS. The procedure may be applied to whole areas to find the best time for the survey campaign. Taking a step further, this approach has been made available as a service by creating a Web Processing Service, which allows also non GIS specialists to access this tool through a simple WebGIS interface.

The user can select the satellites constellation, GPS or GPS and GLONASS, the cut-off elevation angle, the day, hour and temporal window of the survey. The service returns raster maps describing the minimum number of visible satellites and the PDOP index in every pixel. Moreover, the service implements some features of the UNAVCO TEQC software, which can help to choose the location of new permanent stations.

The implementation follows the OGC specifications and relies fully on FOSS software, combining different systems to provide the user web interface, the data storage and the processing engine.

The service features, the underlying technology and some examples will be presented in this paper.
\end{abstract}

\section{INTRODUCTION}

The more and more spread presence of GNSS (Global Navigation Satellite Systems) Permanent Station Networks makes the satellite survey techniques more simpler, quicker and cheaper, allowing the technician to easily obtain high precisions. However, the result and, above all, the speed of GNSS survey campaign get an advantage out of accurate planning, especially where the site to survey has characteristics that make the satellite observations difficult. Most of the GNSS office suites provide planning tools to analyze the satellites visibility. They usually allow planning on single points, taking into account the obstructions due to terrain morphology or buildings by hand-drawing polar diagrams after a visit of each location to be surveyed.

A planning software, integrated in the Free and Open Source GIS GRASS, has been developed to make fully automatic the obstruction determination, by using a digital surface model, and the creation of realistic satellites visibility maps, on whole areas. Taking a step further, the procedure was made available as a service by creating a Web Processing Service, which allows also non GIS specialists to access this tool through a simple WebGIS interface.

\section{GNSS PLANNING}

It is common practice to leave to operator experience and to unobstructed planning the choice of the best time to carry out GNSS measurements, relying the success of the survey on the growing number of satellites available, of both NAVSTAR GPS and GLONASS constellations.

Many of the available commercial software suites allow GNSS planning for individual points; they evaluate the number of visible satellites from a given point over the horizon or over a given elevation angle (cut-off), and provide maps depicting their number and their geometric configuration, the DOP (Dilution of Precision) index, at different time steps during the selected time span (Figure 1).

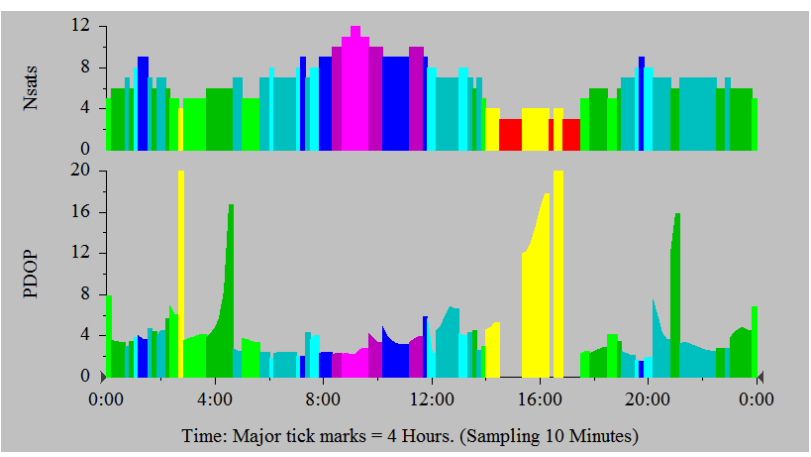

Figure 1: GNSS satellites number and PDOP plots from commercial software

Manual introduction of obstructions and impediments to the sig- 
nal is usually possible but it requires burdensome on-site reconnaissance (Figure 2). Instead, the authors are not aware of software for a planning on large areas.

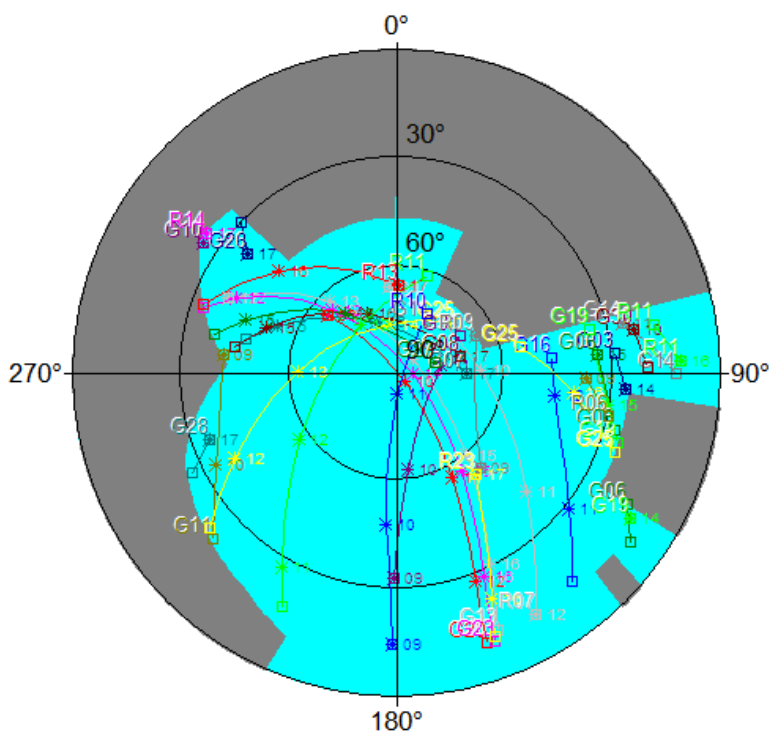

Figure 2: Polar skyplot with hand drawn obstructions

\subsection{GNSS planning with GIS}

A GNSS planning software has been developed (Fruet et al., 1999), (Sguerso and Zatelli, 1999), (Zatelli and D'Incà, 2004) and recently updated (Federici and Sguerso, 2009) as modules of the Free and Open Source GIS GRASS. It allows the identification of optimal areas to perform a survey in a given temporal window, or the best time interval for a survey campaign in a certain area, taking into account the realistic obstructions to satellite signals due to terrain morphology and buildings automatically; moreover, it may be applied to whole areas.

Taking advantage of the GIS environment, the procedure for creating maps of realistic satellites visibility, called realistic planning, involves three logical steps: calculation of the obstructions to satellite signals due to terrain morphology or buildings, determination of the relative position of satellite-receiver and check of the visibility of each satellite, then creation of maps showing the number of visible satellites, PDOP (Positioning Dilution of Precision) index and an index of visibility percentage, in each cell of the analyzed region.

A first module, r.obstruction, determines realistic obstructions on the area from three-dimensional models of the orographic surface and/or building (Digital Surface Models). The obstruction calculation is based on the determination of the maximum elevation of obstacles in the territory; this is done by determining the highest line of sight between all possible lines connecting the observation point at any other point in the DSM along a fixed azimuthal direction. This operation, fully automatic, is performed for each cell scanning for the entire horizon, with azimuth resolution set by the user, creating the polar pattern of obstruction for each point. The heavy computational calculation is counterbalanced by the need to perform this calculation only once, whatever the period in which GNSS planning has to be undertaken, assuming invariance of obstruction in time. The module r.obstruction requires in input the DSM of the area under consideration, the azimuthal resolution and the cut-off angle, which represents the minimum obstruction angle.

A second module, r.planning.static, determines the satellites position at each time from the values of the Keplerian parameters, provided by the almanac file of the available GNSS constellation.
Then the relative positions between satellites and points on the ground is obtained moving from the conventional celestial reference system (CCRS) to a reference system locally defined with respect to the barycentre of the area. The verification of the actual visibility of each satellite is accomplished by comparing azimuth and elevation of obstructions and of satellites at given time. In cells where the number of realistically visible satellites is equal or greater than 4 , the code is also able to evaluate the PDOP index. Thus, realistic maps of satellite visibility and of PDOP are obtained, both evaluated for individual instants or for a time window on whole areas defined by the operator. If queries are carried out for a time window, the results are relative to the worst configuration in each cells in the whole time interval, i.e. corresponding to the minimum number of satellite and to the maximum PDOP index.

This second module has been also integrated with the evaluation of an index of visibility percentage with respect to the number of satellite potentially observable without obstructions, so to help the choice of the site for new GNSS permanent stations. The implementation of such index follows the criteria used by the TEQC software of UNAVCO for GNSS quality control (TEQC toolkit for GNSS data pre-processing, 2010): the percentage of the number of observations actually acquired over the maximum acquirable without obstructions, for the whole day, is assessed with a minimum cut-off angle of 5 degrees. In the current code the evaluation of satellite availability and of number of satellites realistically visible taking into account the obstructions provided by the DSM, is made every five minutes and integrated over 24 hours to finally get an overall percentage for the entire day. Moreover, for a conservative design of permanent station, such estimates were made considering only the GPS constellation.

The module r.planning.static requires in input the extension of analyzed area, the obstructions already determined by the previous module, the GNSS almanac, the starting date and time and the duration of the planning.

\section{WPS FOR GPS PLANNING}

\subsection{OGC WPS}

The pervasive availability of geographic information enables new users to access and use them. However, geographic information is really available to a large group of non specialists users only when easy and intuitive interface to data management and processing are deployed. The current trend in the field of geographic information management is to organize data collection through Sensor Observation Services on one hand and to cater to the users' processing needs using Web Processing Services (WPS) on the other.

Such services, as defined by OGC (Open GIS Consortium Inc., 2007), provide geographic data processing capabilities, usually through a Web or WebGIS interface, across a network. A WPS provides also a standardized interface for the discovery and binding of the geospatial processes by clients.

The complexity of the model the WPS implements varies, but a spatial component of the data is always used. In most of the implementations some of the data are available on the server publishing the WPS and some of the data are provided by the user: usually only simple maps or numerical parameters are requested to the user.

Different implementations of the OGC WPS 1.0.0 standard are available, the most used being FOSS projects, such as 52 North (52 North (52 North Geoprocessing Community), 2010), ZOO Project (ZOO Open WPS platform, 2010), Degree (Deegree Free Software for Spatial Data Infrastructures, 2010) and PyWPS (PyWPS. an implementation of the Web processing Service stan- 
dard from Open Geospatial Consortium, 2010). The latter is used in the application described here.

\subsection{WPS IMPLEMENTATION}

The integration of the WPS in a WebGIS has been carried out using PyWPS (PyWPS. an implementation of the Web processing Service standard from Open Geospatial Consortium, 2010). This choice is justified by the fact that PyWPS natively supports GRASS GIS on one hand, and by the straightforward implementation in the python language of the server side GRASS procedure on the other.

With ease of use in mind, the user is requested to provide only a few input parameters for the execution of the planning procedure. Other parameters are set to default values to simplify and make more intuitive the user interface, choosing a set of values suitable for most of the situations. For example, in the current instance, the cut-off angle is set to 10 degrees and the ellipsoid used to evaluate the center of the area is always Hayford's (also known as "International"), since coordinates are provided in the Italian Gauss-Boaga/Rome40 datum.

The user just chooses the interesting area, with the usual zoom and pan tools available in the WebGIS, the starting day and hour and the duration of the planning, with a dedicated interface, and which satellites' constellation is to be used: GPS only (currently 32 satellites) or GPS + GLONASS (currently $32+24$ satellites) (Figure 3).

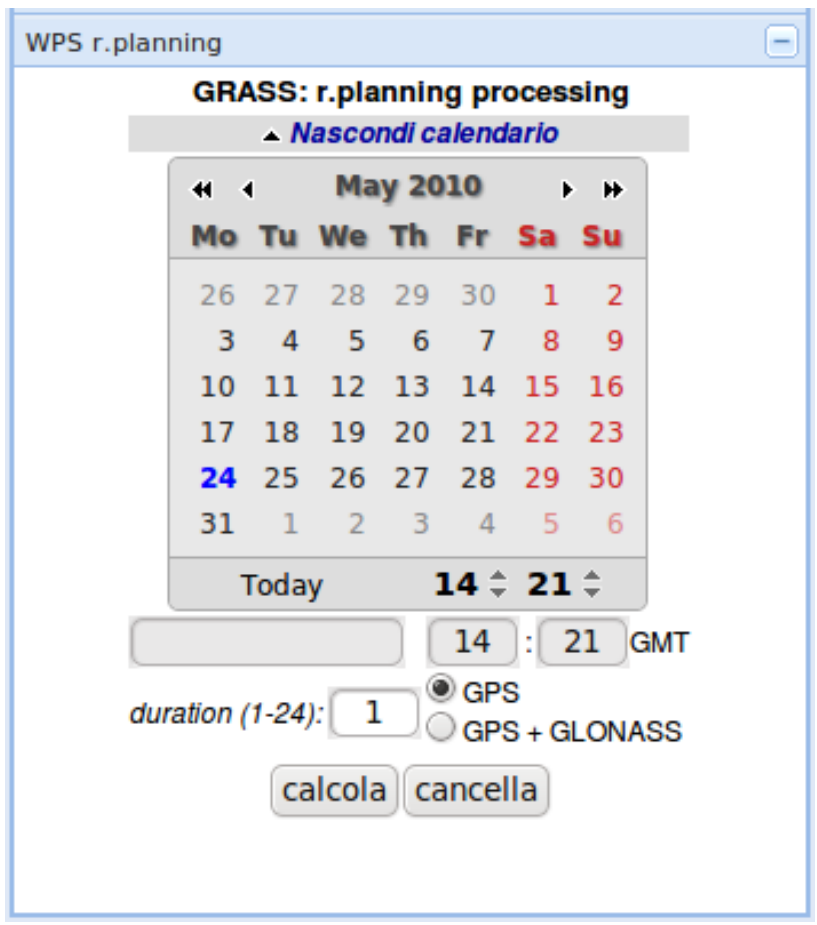

Figure 3: GNSS planning WPS interface

The almanac for the satellites' ephemerides evaluation is automatically downloaded daily from ftp://ftp.trimble.com/pub/eph/. This almanac provides the whole necessary information about the ephemerides for both GPS and GLONASS constellations. Once all the input fields are complete, the process is started with an asynchronous call (thus the WebGIS interface can be used while the planning process runs in the background) to the PyWPS module on the server. The PyWPS module runs a GRASS script inside a temporary GRASS mapset, which is deleted once the procedure is completed. The analysis is performed on the whole area visible when the procedure is started. Two GRASS modules are used sequentially: r.obstruction creates the obstructions file and r.planning.static creates the maps containing the number of visible satellites, the PDOP index and visibility percentage of satellites.

The robstruction module can require long execution times, especially if applied to a wide area. In principle, this module could be executed only once for a wide area: every time an user selects a smaller area the relevant part of the obstruction file should be extracted and used by the r.planning.static module. However, the current implementation of the r.planning.static module is not able to extract subregions from an obstruction file, therefore this file must be created every time according to the current region, selected by the user.

To limit CPU load and running time, therefore the time the user waits for the resulting maps, the resolution is automatically set so that a maximum number of cells has to be evaluated. This obviously means that a low resolution is used when large areas are selected: on the other hand this kind of analysis yields to interesting results only when an area reasonably interested by a satellite survey is selected. Still, the application of the procedure to large areas can provide a reasonable idea of where problems due to a low number of visible satellites can occur, albeit on a low resolution map.

The process returns five output maps: number of visible satellites, at the planning starting time and the minimumn over the planning time span, PDOP index, at the beginning of the planning and the maximum for the time window, and the minimum visibility percentage for the time window. The process output consists of two parts (Figures 4, 5 and 6):

1. five output maps of the r.planning.static module, as ASCIIGRID files, which are available for downloading through a link to a compressed folder containing them;

2. five new temporary layers in the WebGIS, inside their own layer tree, containing the same maps above. These new layers provide a legend to the values of the maps.

For a more immediate interpretation, the maps so produced have been colored as follows:

- for the satellites visibility maps, red pixels represent areas where a GNSS survey is not possible because less than 4 satellites are realistically visible; yellow, green and blue pixels represent areas where the number of satellites is sufficient (4-7 satellites), good (8-11 satellites) or very good (more than 11 satellites) respectively;

- for the PDOP index maps, green pixels represent a good geometric configuration of satellites (PDOP $\leq 3$ ), while yellow and red pixels represent sufficient $(3<\mathrm{PDOP}<7)$ and inadequate (PDOP $\geq 7$ or not calculable if less than 4 satellites are observable) index values respectively;

- for the index of visibility percentage, green pixels are indicative of good satellite visibility (index ranging between $80 \%$ and $100 \%$ ), hence of sites suitable for the installation of new GNSS permanent stations; yellow and red pixels correspond to percentages respectively between $70 \%$ and $80 \%$ and less than $70 \%$.

\section{TESTS}

Planning results from the WPS have been verified by comparing the number of visible satellites to the number of satellites actually 
tracked by 8 GPS permanent stations in the Trentino region in Italy. These permanent stations constitute the regional network for real time positioning (TPOS, Figure 7) in the Autonomous Province of Trento, in northern Italy.

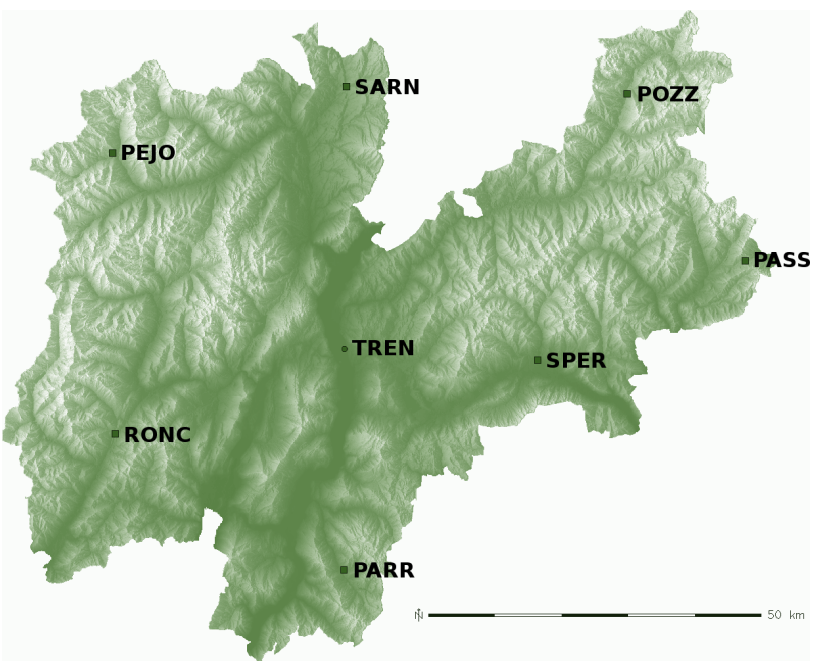

Figure 7: TPOS permanent stations network

While the locations of the permanent stations have been obviously selected to provide the minimum obstacle to sky view, the area is mountainous, therefore the number of satellites visible can be substantially lower than that predicted by the almanac without taking into account obstructions.

The number of visible satellites obtained from the maps created by the WPS has been compared to the numbers of satellites tracked by the 8 stations (RNX, in Table 1), which has been verified by checking the RINEX files for each station.

The test has been carried out on two time windows of 1 hour each, 10-11 and 15-16 GMT+00, on June, the 3rd, 2010. The minimum number of visible satellites in each hour is used. DTM resolution is 40 meters.

Table 1 shows the results of the test. Here WPS indicates the number of satellites provided by the WPS model and RNX the number of observed satellites evaluated from the RINEX files.

The symbol "=" denotes that the number of visible satellites is the same for WPS and RINEX, "+" means that the number of satellites is higher for th RINEX and "-" that the number of satellites is lower for the RINEX.

\begin{tabular}{|c|c|c|c||c|c|c|}
\hline & \multicolumn{3}{|c||}{$10-11$ GMT+00 } & \multicolumn{3}{c|}{ 15-16 GMT+00 } \\
\hline \hline Station & WPS & RNX & & WPS & RNX & \\
\hline PASS & 6 & 6 & $=$ & 5 & 7 & + \\
\hline PARR & 5 & 5 & $=$ & 7 & 7 & $=$ \\
\hline PEJO & 7 & 6 & - & 5 & 6 & + \\
\hline POZZ & 6 & 7 & + & 6 & 7 & + \\
\hline RONC & 6 & 6 & $=$ & 6 & 6 & $=$ \\
\hline SARN & 6 & 7 & $=$ & 8 & 8 & $=$ \\
\hline SPER & 7 & 7 & $=$ & 8 & 8 & $=$ \\
\hline TREN & 7 & 7 & $=$ & 7 & 8 & + \\
\hline
\end{tabular}

Table 1: Test results: number of visible GPS satellites (June, the 3rd, 2010), predicted (WPS) and observed (RNX)

Out of a total number of 16 samples ( 8 stations for two time windows), 10 show a perfect accord between predicted (using the WPS) and observed (RINEX) number of satellites, in 5 cases the number of visible satellites is higher than predicted and only in 1 case (PEJO station for 10-11 GMT+00) it is lower than predicted. It must be noted that, with the only exception of the PASS station for the 15-16 GMT+00 time window, the difference is of just one satellite.

Tests show a good agreement between the number of predicted and observed satellites. The small differences can be explained by:

1. the relatively low resolution used for the DTM (40 m);

2. the limited area used to evaluate obstructions, which can leave out nearby mountains representing actual obstructions (tests have been carried out using an approximately $4 \times 3 \mathrm{~km}$ area);

3. the use of a DTM instead of a DSM, which can make some difference for stations in populated areas, where tall buildings can hide part of the sky.

However, results for the TREN station, located in the outskirt of the city, contradict this latter hypothesis, predicting a lower than observed number of visible satellites, even when a DTM instead of a DSM is used. This is probably due to the second effect listed above, with relatively high mountains south of the GPS station and outside the region used for the planning. The only way to remove this effect is to select large areas for the planning, with the tradeoff of obtaining low resolution maps, discarding maps' borders.

Most importantly, except for one case, all the estimations are conservative in predicting lower than observed numbers of satellites. Figure 8 shows the comparison between a traditional GPS satellites plot for survey planning (without taking into account obstructions) and the number of satellites actually observed on the site for the PARR permanent station in the 15-16 GMT+00 time window; the number of visible satellites from the almanac is on the background (pink, yellow and red areas), while the number from the RINEX file is in the foreground (in green and blue).

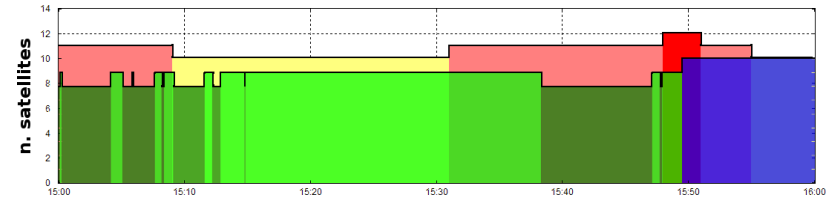

Figure 8: Number of visible satellites for the PARR permanent station in the 15-16 GMT+00 time window, from the almanac on the background and from the RINEX file in the foreground

Without taking into account obstructions, the prediction of the number of visible satellites from a commercial software in Figure 8 is optimistic, while the prediction for the PARR station from the WPS in Table 1 is accurate.

An additional test has been carried out using the robstruction module directly in GRASS to evaluate the sky obstructions and the TEQC (TEQC toolkit for GNSS data pre-processing, 2010) software to estimate the number of visible satellites, comparing it to the number of observed satellites (Figure 9).

In Figure 9 red arcs represent the parts of the satellites' trajectories not observed by the station (therefore the corresponding GPS measurements data are not available in the RINEX file), while grey areas correspond to obstacles: there is a good correspondence between these parts of the trajectories and the obstructions evaluated by the robstruction module.

\section{CONCLUSIONS AND FUTURE WORK}

The WPS service for GNSS realistic planning has passed the testing phase and it is now used routinely. Its implementation in other 


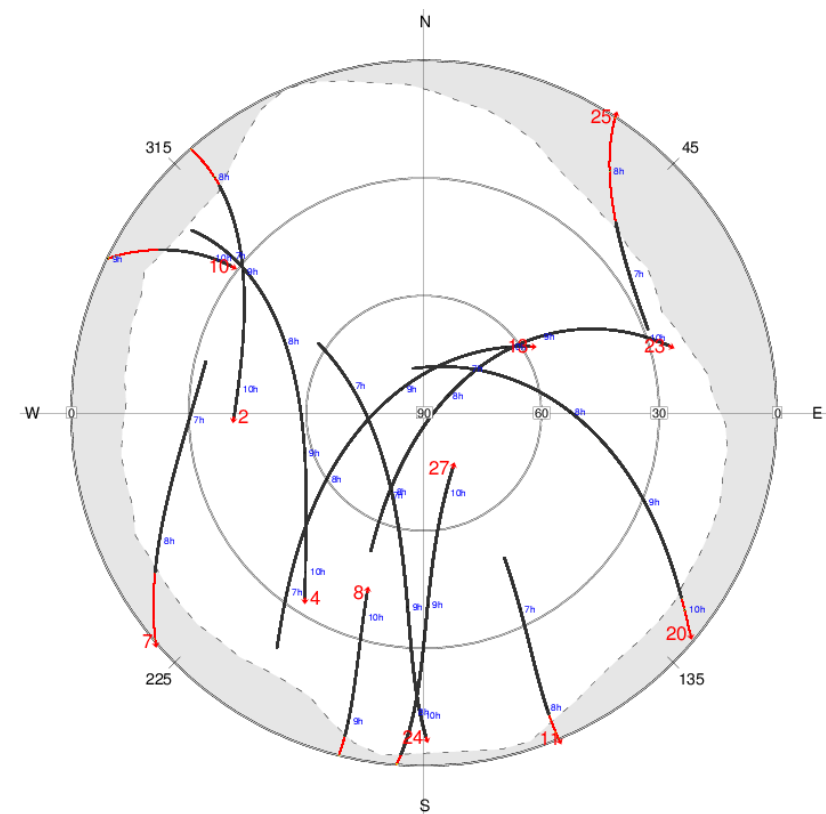

Figure 9: Skyplot for the TREN site, with obstruction from GRASS's robstruction module; red archs represent the parts of the satellites' trajectories not observed by the station, grey areas correspond to obstacles

WebGIS services covering other Italian regions is under way. The sequence of the two GRASS modules implemented in the WPS is very useful for an optimum choice of time intervals and of the organization of measurement sessions in complex areas. Tests indicate a good reliability of the prediction of the number of satellites resulting from the WPS output maps; when the numbers of visible satellites do not agree, the difference is small and the prediction is conservative. Additional tests have been planned, involving scattered points, different from permanent stations, where obstructions can be more problematic in terms of reduction of the number of visible satellites. The accuracy of the evaluation of the PDOP index by the r.planning.static module has been already evaluated in independent tests.

Future work will consist in:

- the use of the Shuttle Radar Topography Mission (SRTM) digital elevation model (Official NASA SRTM site, 2010) or the ASTER Global Digital Elevation Model (ASTER Global Digital Elevation Map. NASA, 2010), extending the availability of the WPS to all the regions where these DTMs are available (from $56^{\circ} \mathrm{S}$ to $60^{\circ} \mathrm{N}$ for the SRTM dataset, from $83^{\circ} \mathrm{S}$ to $83^{\circ} \mathrm{N}$ for the ASTER dataset); the application of the procedure should be accurate even for areas where only lower resolution (three-arc-second, approx. 90-meter) data are available if the SRTM dataset is used, the ASTER 30 meters resolution is higher than the one used in our tests;

- the implementation of a "multi-station network" mode to predict the number of common visible satellites for a set of permanent stations and a position selected by the user;

- the implementation of an additional procedure to assess the variability of the estimated parameters (number of visible satellites and PDOP) within the time span of the planning;

- the implementation of the WPS for other datums than the Italian Gauss-Boaga/Rome40 one and the possibility of taking into account the local time offset to the GMT in the user interface of the WPS, making its use more intuitive for the user;

- the possibility to evaluate satellites' visibility for single 2D (on a DTM or DSM) or 3D points or lines.

\section{ACKNOWLEDGEMENTS}

This research is partially founded by Ministero dell'Istruzione, dell'Università e della Ricerca (MIUR) within the MIUR-COFIN 2007 "Interoperability and cooperative management of geographic, dynamic, multidimentional and distributed data with Free and Open Source GIS" project.

\section{REFERENCES}

52 North (52 North Geoprocessing Community), 2010. http://52north.org/wps (accessed 18 May 2010).

ASTER Global Digital Elevation Map. NASA, 2010. http://asterweb.jpl.nasa.gov/gdem.asp (accessed 18 May 2010).

Deegree - Free Software for Spatial Data Infrastructures, 2010. http://www.deegree.org/ (accessed 18 May 2010).

Federici, B. and Sguerso, D., 2009. Planning GNSS realistico per applicazioni statiche e cinematiche. Bollettino SIFET sessione scientifica, ISSN: 1721-971X 1(1), pp. 79-93.

Fruet, G., Sguerso, D. and Zatelli, P., 1999. Planning GPS automatico con ostruzioni realistiche. In: Atti del convegno: "3a Conferenza nazionale delle Associazioni Scientifiche per le Informazioni Territoriali ed Ambientali”, Napoli.

Official NASA SRTM site, 2010. http://www2.jpl.nasa.gov/srtm/ (accessed 18 May 2010).

Open GIS Consortium Inc., 2007. OpenGIS Web Processing Service Implementation Specication, OpenGIS Standard 05-007r7. $\mathrm{http} / / /$ www.opengeospatial.org/standards/wps (accessed 18 May 2010).

PyWPS. an implementation of the Web processing Service standard from Open Geospatial Consortium, 2010. http://pywps.wald.intevation.org/ (accessed 18 May 2010).

Sguerso, D. and Zatelli, P., 1999. Planning of kinematic GPS including realistic obstacles description. In: XXII General Assembly of IUGG, Birmingham, UK

TEQC toolkit for GNSS data pre-processing, 2010. UNAVCO: http://facility.unavco.org/software/teqc/teqc.html (accessed 18 May 2010).

Zatelli, P. and D'Incà, D., 2004. New modules for satellite surveying planning in GRASS. Geomatics Workbooks 3, pp. 1-14.

ZOO Open WPS platform, 2010. http://www.zoo-project.org/ (accessed 18 May 2010). 


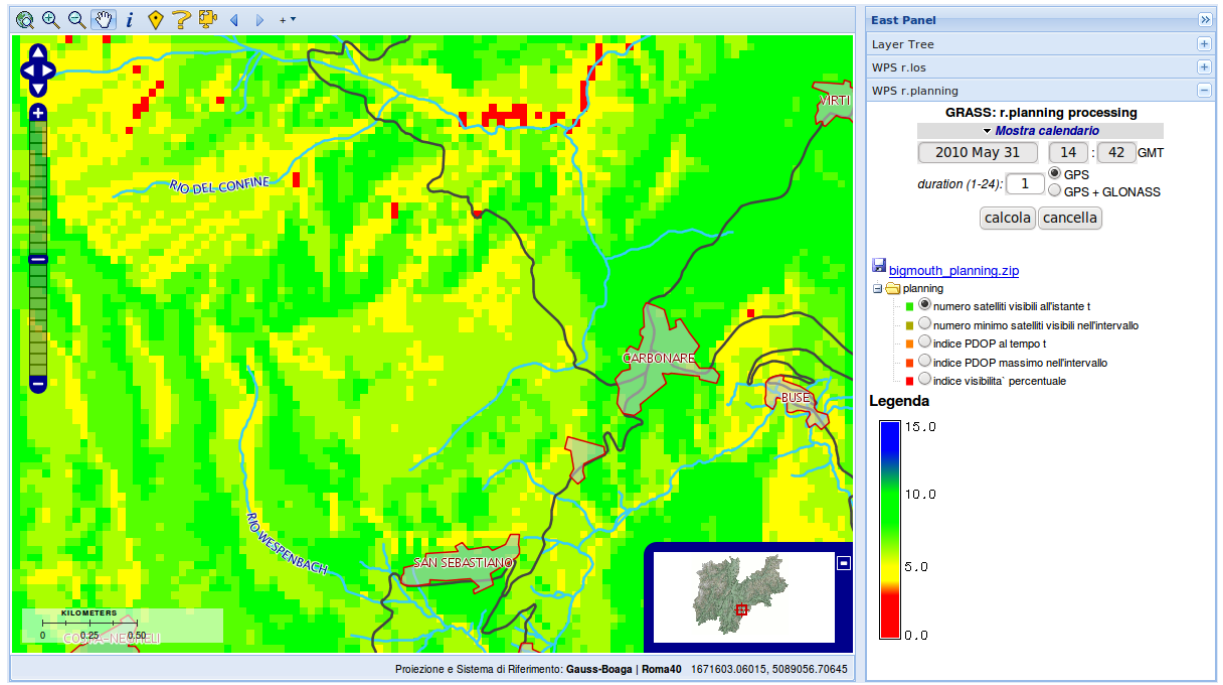

Figure 4: GNSS planning WPS output and download: number of visible satellites

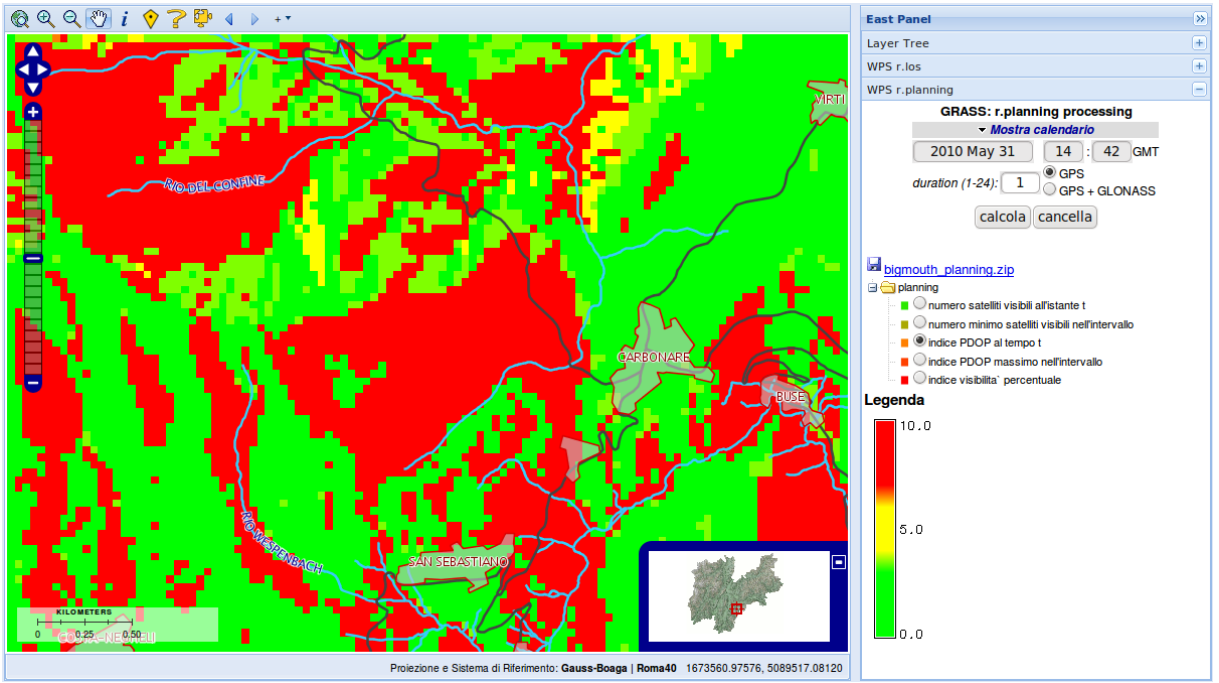

Figure 5: GNSS planning WPS output and download: PDOP

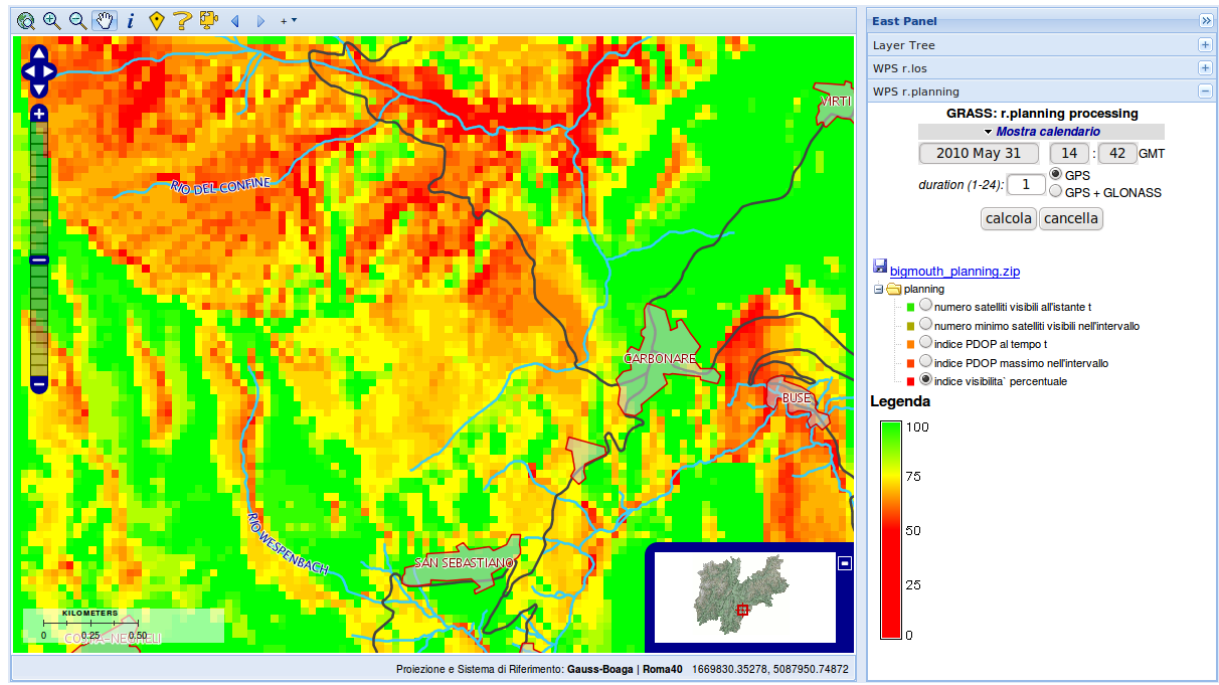

Figure 6: GNSS planning WPS output and download: \% of visible satellites with respect to the maximum available without obstructions 